\title{
FOXP3 Allelic Variants and Haplotype Structures Are Associated with Aggressive Breast Cancer Subtypes
}

\author{
Bruna Karina Banin Hirata, ${ }^{1}$ Roberta Losi Guembarovski, ${ }^{2}$ Glauco Akelinghton Freire Vitiello, ${ }^{1}$ \\ Alda Losi Guembarovski, ${ }^{3}$ Karen Brajão de Oliveira, ${ }^{1}$ and Maria Angelica Ehara Watanabe \\ ${ }^{1}$ Department of Pathological Sciences, Biological Sciences Center, State University of Londrina, Celso Garcia Cid Highway, \\ Pr 445 Km 380, Campus Universitário, 86057-970 Londrina, PR, Brazil \\ ${ }^{2}$ Department of General Biology, Biological Sciences Center, State University of Londrina, Celso Garcia Cid Highway, Pr 445 Km 380 , \\ Campus Universitário, 86057-970 Londrina, PR, Brazil \\ ${ }^{3}$ Department of Pathology, Clinical and Toxicological Analysis, Health Science Center, State University of Londrina, \\ Celso Garcia Cid Highway, Pr 445 Km 380, Campus Universitário, 86057-970 Londrina, PR, Brazil
}

Correspondence should be addressed to Maria Angelica Ehara Watanabe; maewatuel@gmail.com

Received 5 April 2017; Accepted 24 May 2017; Published 21 June 2017

Academic Editor: Leigh A. Madden

Copyright (c) 2017 Bruna Karina Banin Hirata et al. This is an open access article distributed under the Creative Commons Attribution License, which permits unrestricted use, distribution, and reproduction in any medium, provided the original work is properly cited.

\begin{abstract}
FOXP3 genetic polymorphisms have been associated with cancer development and prognosis. In this context, the present study aimed to evaluate the g.10403A $>\mathrm{G}$ (rs2232365) polymorphisms and g.8048A $>\mathrm{C}$ (rs3761548), in aggressive breast cancer (BC) subtypes, including, Luminal B HER2+ (LB), HER2-enriched (HER2+), and triple-negative (TN). Polymerase chain reaction followed by enzymatic restriction was performed to genotyping $117 \mathrm{BC}$ samples and 300 controls. A significant association of AA genotype $(\mathrm{g} .10403 \mathrm{~A}>\mathrm{G})$ in relation to $\mathrm{BC}$ susceptibility $(\mathrm{OR}=1.93 ; 95 \% \mathrm{CI}=1.01-3.66$; $p=0.046)$ was observed. The GG (g.10403A $>$ G) genotype was correlated with higher proliferation index (Ki-67) in HER2+ subtype $(\tau=0.47 ; p=0.019)$ and advanced TNM staging in TN $(\tau=0.23 ; p=0.032)$. A correlation of AA genotype (g.8048A $>$ C) with higher Ki-67 $(\tau=-0.47 ; p=0.018)$ and lower histological grade $(\tau=0.39 ; p=0.026)$ in HER2+ was also found. GA haplotype was correlated with lower histological grade $(\tau=-0.15 ; p=0.009)$ and higher Ki-67 ( $\tau=0.43$; $p=0.036)$ in HER2+ and advanced staging in TN $(\tau=0.29 ; p=0.044)$. On the other hand, AC haplotype was correlated with lower Ki-67 $(\tau=-0.54 ; p=0.005)$ and staging $(\tau=-0.29 ; p=0.027)$ in HER2+ and TN respectively. Results showed that FOXP3 influence regarding clinical outcome depends greatly on the BC subtype and indicated this transcription factor as a promising marker in aggressive $\mathrm{BC}$ subtypes.
\end{abstract}

\section{Introduction}

The National Cancer Institute (INCA) estimated 57,960 new cases of breast cancer (BC) for 2016 and 2017 in Brazil. It is worth noting that, regardless of nonmelanoma skin cancer, the mammary tumor is the most common among women in many regions of the country, accounting for high morbidity and mortality [1].

$\mathrm{BC}$ represents a complex and heterogeneous disease that comprises distinct pathologies, histological features, and clinical outcome. The status of estrogen receptor
(ER), progesterone receptor (PR), human epidermal growth factor receptor type 2 (HER2), and proliferation index Ki-67 has been used as predictive markers to identify high-risk phenotypes and for selection of most efficient therapies [2-4].

These molecular markers are also generally used to define $\mathrm{BC}$ subtypes, namely Luminal A (LA; ER/PR ${ }^{+} \mathrm{HER} 2^{-}$), Luminal B (LB; ER/PR ${ }^{+} \mathrm{HER} 2^{+}$or ER/PR ${ }^{+} \mathrm{HER} 2^{-} \mathrm{Ki}-67^{\mathrm{High}}$ ), HER2-enriched (HER2+; ER/PR ${ }^{-} \mathrm{HER} 2^{+}$), and basal-like, also termed as triple-negative (TN; ER/PR ${ }^{-} \mathrm{HER} 2^{-}$) [5]. Among these subtypes, the basal-like has the worst prognosis, 
while Luminal A has the best [6]. Within tumors that present HER2 overexpression, Luminal B (hormonal receptors positive) was associated with better prognosis compared with the HER2-enriched subtype [7].

Forkhead box P3 (FOXP3) is an essential transcription factor to the development and functions of Regulatory T cells (Tregs) [8]. Increased levels of $\mathrm{FOXP}^{+}$Tregs in peripheral blood and tumor microenvironment have been reported in diverse cancer types, including the breast one [9]. These cells play an important role in immune response suppression and thus may contribute to tumorigenesis.

The accumulation of Tregs in local lymph nodes or in tumors is associated with an unfavorable prognosis $[10,11]$. Although Tregs are the major cell type expressing FOXP3, it has been demonstrated that tumor cells themselves can express this protein, such as those in pancreatic cancer [12], melanoma [13], and breast tumors [14, 15]. Moreover, FOXP3 expression in tumor cells could be an independent strong prognostic factor for distant metastasis in BC [16]. However, in contrast with this data, this transcription factor was also shown to be a tumor suppressor gene, acting as a transcriptional repressor of SKP2 and HER2, two important BC oncogenes $[17,18]$.

Considering FOXP3 dual role in tumor microenvironment, investigation of polymorphisms and their possible associations with cancer may shed light on the molecular cancer pathogenesis and open new perspectives to susceptible individual screening [19].

Polymorphisms in the FOXP3 gene may change its product quantitatively or functionally, thereby contributing to an immune imbalance in cancer. To date, FOXP3 allelic variants have been associated with a variety of immune-related diseases, such as allergic rhinitis [20], idiopathic infertility, and endometriosis-related infertility [21]. Furthermore, FOXP3 polymorphisms have also been associated with different types of cancer, such as Wilm's tumor [22], hepatocellular carcinoma [23], colorectal cancer [24], and nonsmall cell lung carcinoma [25]. However, few studies have investigated BC patients $[26,27]$, especially in their molecular subtypes and in relation to their clinical outcomes.

In this context, the present study aimed to investigate possible association between two FOXP3 single nucleotide polymorphisms (SNPs) regarding susceptibility and clinical outcome in aggressive BC subtypes (LB, HER2+, and TN) from a South Brazilian sample.

\section{Materials and Methods}

2.1. Ethical Aspects and Sample Characterization. Patients and controls were informed in detail regarding the research, and the consent term was obtained. In the present study, 107 peripheral blood samples $(5 \mathrm{~mL})$ collected with EDTA as anticoagulant and 10 paraffin-embedded tissues from patients attended in the Cancer Hospital of Londrina, Londrina, Paraná, Brazil (CHL) were included. In total, 117 BC samples were obtained, of which 37 were diagnosed as Luminal B HER2+ (LB), 26 as HER2-enriched (HER2+), and 54 as triple-negative (TN) subtypes.
For the control group, 300 blood samples were collected from women of same geographic region, without BC, proved by clinical and imaging examination, no self-declared $\mathrm{BC}$ family history or personal history of any malignant disease.

Clinicopathologic parameters data and immunohistochemical classification of BC subgroups were retrieved from patients' medical register available at CHL. Prognostic parameters included tumor size, lymph node commitment, proliferation index Ki-67, histological grade, and clinicopathological staging (Tumor/Node/Metastasis classification), which were determined according to the Union of International Control of Cancer classification criteria [28].

2.2. Genomic DNA Extraction. Genomic DNA was obtained from peripheral blood cells using Biopur Mini Spin Plus Kit (Biometrix Diagnostica, Curitiba, Brazil), according to the manufacturer's instructions. From the formalin fixed and paraffin-embedded samples, DNA was extracted using innuPREP DNA Mini (Analytik Jena, Jena, Germany), according to manufacturer's protocol. All samples were quantified by NanoDrop 2000c ${ }^{\circledR}$ Spectrophotometer (Thermo Scientific, Wilmington, USA) at a wavelength of $260 / 280 \mathrm{~nm}$, and the final preparations were stored at $-20^{\circ} \mathrm{C}$.

2.3. FOXP3 Genotyping. Polymerase chain reaction (PCR) followed by enzymatic restriction (PCR-RLFP) was performed to genotype rs2232365 and rs3761548 SNPs (HGVS names: g.10403A > G and g.8048AC, resp., according to Gen Bank Accession number NG_007392.1).

For g.10403A $>\mathrm{G}$ genotyping, the following primers were used: $5^{\prime}$-AGGAGAAGGAGTGGGCATTT-3 ${ }^{\prime}$ (forward) and 5'-TGTGAGTGGAGGAGCTGAGG-3' (reverse), according to Paradowska-Gorycka, Jurkowska [29]. The g.8048A>C genotyping was performed with the following primers: 5'-GGCAGAGTTGAAATCCAAGC-3' (forward) and 5'-CA ACGTGTGAGAAGGCAGAA-3' (reverse), according to He et al. [25]. The PCR was conducted using $1 \mathrm{X}$ of PCR Buffer (20 mM of Tris- $\mathrm{HCl}$ ph $8.5 ; 50 \mathrm{mM}$ of $\mathrm{KCl}$ ), $0.8 \mathrm{mM}$ of $\mathrm{MgCl}_{2}, 0.1 \mathrm{mM}$ of $\mathrm{dNTP}, 0.2 \mu \mathrm{M}$ of each primer, $0.05 \mathrm{U} / \mu \mathrm{L}$ of Taq DNA polymerase, and $4 \mathrm{ng} / \mu \mathrm{L}$ of genomic DNA diluted in ultra-pure $\mathrm{H}_{2} \mathrm{O}$ (Milli-Q) to complete a final volume of $25 \mu \mathrm{L}$ per reaction tube. Negative controls were employed to make sure that no contaminants were introduced. The cycling protocol, used to both FOXP3 polymorphisms, was a denaturation at $94^{\circ} \mathrm{C}$ for $5 \mathrm{~min}, 35$ cycles of $45 \mathrm{sec}$ at $94^{\circ} \mathrm{C}, 45 \mathrm{sec}$ at $59^{\circ} \mathrm{C}$ to g. $10403 \mathrm{~A}>\mathrm{G}$ or $65^{\circ} \mathrm{C}$ to g. $8048 \mathrm{~A}>\mathrm{C}, 45 \mathrm{sec}$ at $72^{\circ} \mathrm{C}$, and $10 \mathrm{~min}$ of final elongation at $72^{\circ} \mathrm{C}$. PCR products $(5 \mu \mathrm{L})$ of $\mathrm{g} .10403 \mathrm{~A}>\mathrm{G}$, with $249 \mathrm{bp}$, were digested overnight at $55^{\circ} \mathrm{C}$ with 1 unit/ reaction of BsmBI restriction endonuclease (New England Biolabs, Beverly, USA), generating two fragments of $132 \mathrm{bp}$ and $117 \mathrm{bp}$ corresponding to allele G. The PCR products $(6 \mu \mathrm{L})$ of g.8048A $>\mathrm{C}$, with $155 \mathrm{bp}$, were digested overnight at $37^{\circ} \mathrm{C}$ with 2 units/reaction of PstI restriction endonuclease (New England Biolabs, Beverly, USA), generating two fragments of $80 \mathrm{bp}$ and $75 \mathrm{bp}$ that correspond to allele C. All PCR and digested products were analyzed on polyacrylamide gel $(10 \%)$, stained with silver nitrate. 
TABLE 1: Prognostic parameters in total BC sample and in aggressive subtypes.

\begin{tabular}{|c|c|c|c|c|c|}
\hline Prognostic parameters & & Total BC & LB & HER2+ & $\mathrm{TN}$ \\
\hline \multirow{3}{*}{ Tumor size } & $<1.5 \mathrm{~cm}$ & $10(8.9 \%)$ & $3(8.3 \%)$ & $2(8.7 \%)$ & $5(9.4 \%)$ \\
\hline & $1.5-3.0 \mathrm{~cm}$ & $57(50.9 \%)$ & $23(63.9 \%)$ & $14(60.9 \%)$ & $20(37.8 \%)$ \\
\hline & $>3.0 \mathrm{~cm}$ & $45(40.2 \%)$ & $10(27.8 \%)$ & $7(30.4 \%)$ & $28(52.8 \%)$ \\
\hline \multirow{4}{*}{ TNM staging } & I & $19(18.1 \%)$ & $7(20 \%)$ & $5(19.2 \%)$ & $7(15.9 \%)$ \\
\hline & II & $39(37.2 \%)$ & $14(40 \%)$ & $8(30.8 \%)$ & $17(38.6 \%)$ \\
\hline & III & $37(35.2 \%)$ & $12(34.3 \%)$ & $9(34.6 \%)$ & $16(36.4 \%)$ \\
\hline & IV & $10(9.5 \%)$ & $2(5.7 \%)$ & $4(15.4 \%)$ & $4(9.1 \%)$ \\
\hline \multirow{2}{*}{ Histological grade } & II & $30(26.8 \%)$ & $12(33.3 \%)$ & $8(34.8 \%)$ & $10(18.9 \%)$ \\
\hline & III & $82(73.2 \%)$ & $24(66.7 \%)$ & $15(65.2 \%)$ & $43(81.1 \%)$ \\
\hline \multirow{3}{*}{$\mathrm{Ki}-67$} & Low & $7(8.8 \%)$ & $4(19.0 \%)$ & $0(0.0 \%)$ & $3(7.3 \%)$ \\
\hline & Moderate & $25(31.2 \%)$ & $9(42.9 \%)$ & $7(46.7 \%)$ & $9(23.6 \%)$ \\
\hline & High & $48(60.0 \%)$ & $8(38.1 \%)$ & $8(53.3 \%)$ & $32(69.1 \%)$ \\
\hline \multirow{2}{*}{ Lymph nodes commitment } & No & $54(49.1 \%)$ & $19(52.8 \%)$ & $12(52.2 \%)$ & $23(45.1 \%)$ \\
\hline & Yes & $56(50.9 \%)$ & $17(47.2 \%)$ & $11(47.8 \%)$ & $28(54.9 \%)$ \\
\hline
\end{tabular}

LB: Luminal B HER+; HER2+: HER2-enriched; TN: triple-negative.

2.4. Haplotype Analysis. FOXP3 haplotypes were determined based on the genotypes of all study participants using PHASE software version 2.1.1 [30, 31]. Permutation test was also performed, using the same software, to check for haplotype distribution differences among controls and BC subgroups.

2.5. Statistical Analysis. Binary logistic regression analyses were conducted to investigate possible associations between polymorphisms or haplotype structures and BC, controlled by age. Associations were tested considering genotypic models (heterozygotes or variant homozygotes versus wild homozygotes), dominant model (heterozygotes and variant homozygotes versus wild homozygotes), and recessive model (variant homozygotes versus wild homozygotes and heterozygotes). In the association study of FOXP3 haplotypes, the following models were analyzed: AC dominant (AA, GC, and GA carriers versus AC carriers), AC recessive (AA, GC, and GA carriers versus ACAC), AA dominant (AC, GC, and GA carriers versus AA carriers), GC dominant (AC, AA, and GA carriers versus GC carriers), GC recessive (AC, AA, and GA carriers versus GCGC), GA dominant (AC, AA, and GC carriers versus GA carriers), and GA recessive ( $\mathrm{AC}, \mathrm{AA}$, and $\mathrm{GC}$ carriers versus GAGA). The AA dominant model was not analyzed in TN subtype because the group did not present this haplotype.

Correlations between polymorphisms or haplotype structures and clinical parameters were assessed by Kendall's tau-b rank correlation coefficient.

All statistical analyses were performed in software SPSS 22.0 version (SPSS Inc., Chicago, USA) and were two-tailed, with $5 \%$ significance level.

\section{Results}

In the present study, the median age of $\mathrm{BC}$ patients was $51( \pm 14)$ years and of control group was $55( \pm 13)$ years $(p=0.118)$. The prognostic parameters in general BC patients and in different subtypes are shown in Table 1. Some parameters were not available.

Eletrophoretic profiles of FOXP3 polymorphisms are shown in Figure 1. Genotype distribution, allele, and haplotype frequencies for both polymorphisms are showed in Table 2. The minor allele frequency (MAF) of g.10403A $>\mathrm{G}$ and g.8048A $>C$ was consistent with the corresponding frequencies reported in 1000 Genomes project (https://www.ncbi.nlm.nih. gov/variation/tools/1000genomes/).

In relation to FOXP3 haplotypes, the predominant was the $\mathrm{AC}$, both in controls and in all BC subgroups, while the less common was the AA. The haplotype frequencies from controls were compared with African, European, American, and Asian populations, using the publicly available data from the 1000 genome project obtained through web-based application LDlink [32]. The haplotype frequencies were significantly different from these populations $\left(p<0.05\right.$ by $\chi^{2}$ test). No significant difference was found in haplotype distribution between controls and $\mathrm{BC}$ patients in the general sample $(p=0.52)$.

In the present study, in the total sample, AA genotype of g.10403A $>\mathrm{G}$ was associated with BC susceptibility $(\mathrm{OR}=1.93 ; 95 \% \mathrm{CI}=1.01-3.66 ; p=0.046)$. No association was found to dominant (GG versus $(A G+A A)$ ) or recessive $((G G+A G)$ versus AA) models. Also, no association with BC susceptibility was found to g.8048A >C polymorphism, in genotype, dominant, or recessive models.

Therefore, no significant association between haplotypes and BC susceptibility was found, both in total sample or in different subtypes. Although, a strong tendency of association of AC haplotype with $\mathrm{BC}$ protection, in total sample in the recessive model $(\mathrm{OR}=0.58$; 95\% $\mathrm{CI}=0.33$ 1.01; $p=0.053)$, and $\mathrm{TN}$ subtype in dominant model $(\mathrm{OR}=0.55 ; 95 \% \mathrm{CI}=0.28-1.07 ; p=0.08)$ was observed. Also, a tendency was found between AA haplotype, in dominant model, and BC protection in $\mathrm{LB}$ subtype $(\mathrm{OR}=0.19 ; 95 \%$ $\mathrm{CI}=0.03-1.05 ; p=0.06)$. 


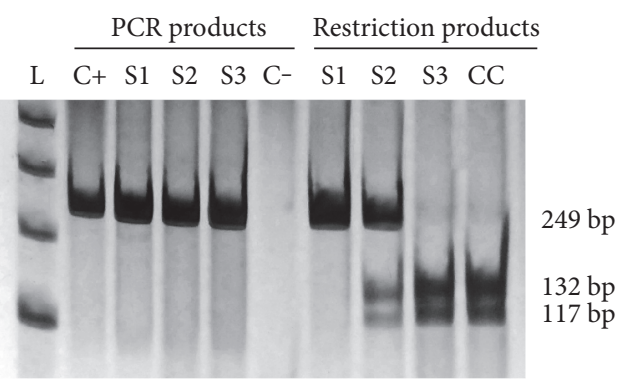

(a)

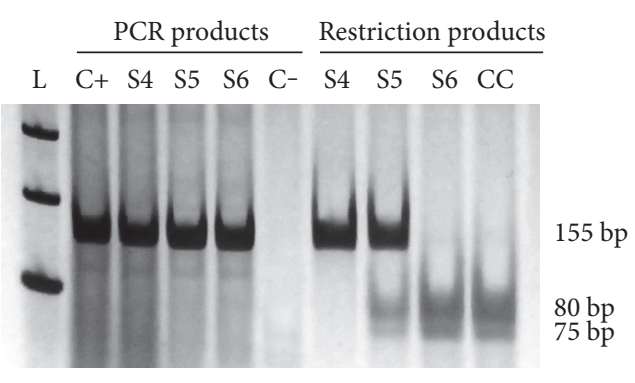

(b)

FIGURE 1: Eletrophoretic profiles of FOXP3 polymorphisms. (a) Eletrophoretic profiles of g.10403A $>$ G (rs2232365). (b) Eletrophoretic profiles of g.8048A>C (rs3761548). L: Ladder 100 bp; C+: positive control; C-: negative control; CC: cleavage control; S1: homozygote genotype AA; S2: heterozygote genotype AG; S3: homozygote genotype GG; S4: homozygote genotype AA; S5: heterozygote genotype AC; S6: homozygote genotype CC.

TABLE 2: Allelic, genotypic, and haplotype frequencies of FOXP3 polymorphisms in total BC sample and in aggressive subtypes.

\begin{tabular}{lccccc}
\hline & Genotype & $\begin{array}{c}\text { Controls } \\
(n=300)\end{array}$ & $\begin{array}{c}\text { Total BC } \\
(n=117)\end{array}$ & $\begin{array}{c}\text { LB } \\
(n=37)\end{array}$ & $\begin{array}{c}\text { HER2+ } \\
(n=26)\end{array}$ \\
\hline & AA & $47(15.7 \%)$ & $26(22.2 \%)$ & $9(24.3 \%)$ & $5(19.2 \%)$ \\
$(n=54)$
\end{tabular}

LB: Luminal B HER2+; HER2+: HER2-enriched; TN: triple negative.

The analysis considering clinical parameters showed a significant correlation between GG genotype of g.10403A>G polymorphism and higher proliferation index $\mathrm{Ki}-67$ in HER2 + subtype $(\tau=0.47 ; p=0.019)$ and advanced TNM staging in TN subtype $(\tau=0.23 ; p=0.032)$. A significant correlation of AA genotype of g.8048A $>C$ polymorphism with higher Ki-67 $(\tau=-0.47 ; p=0.018)$ and lower histological grade, in HER2+ subtype $(\tau=0.39 ; p=0.026)$ (Table 3$)$, was also found.

Furthermore, a significant correlation of GA haplotype with lower histological grade $(\tau=-0.15 ; p=0.009)$ and higher Ki-67 ( $\tau=0.43$; $p=0.036)$ in HER2+ subtype and with advanced staging in TN $(\tau=0.29 ; p=0.044)$ was found. The AC haplotype was correlated with lower Ki-67 ( $\tau=-0.54$; $p=0.005)$ and TNM staging $(\tau=-0.29 ; p=0.027)$ in HER $2+$ and TN subtypes, respectively (Table 4 ).

\section{Discussion}

In the present study, FOXP3 g.10403A $>$ G and g.8048A $>C$ polymorphisms were analyzed in $117 \mathrm{BC}$ patients and 300 neoplasia-free controls. Present results indicated a significant association of AA homozygous genotype (g.10403A>G) in relation to $\mathrm{BC}$ susceptibility $(\mathrm{OR}=1.93,95 \% \mathrm{CI}=1.01$ to 3.66), suggesting that individuals who had inherited both copies of the allelic variant are more susceptible for $\mathrm{BC}$ development than wild homozygous (GG) individuals.

To our knowledge, there are no studies reporting any significant association between g.10403A $>\mathrm{G}$ and $\mathrm{BC}$ susceptibility, but significant associations have been proposed with other diseases, such as psoriasis vulgaris (MAF: allele $G$, cases $=0.19$; controls $=0.27$ ) [33], vitiligo (MAF: allele $\mathrm{G}$, cases $=0.34$; controls $=0.28$ ) [34], unexplained recurrent spontaneous abortion (MAF: allele A, cases $=0.29$; controls $=0.40) \quad$ [35], idiopathic recurrent pregnancy loss (MAF: allele G, cases $=0.40$; controls $=0.29$ ) [36], and autism spectrum disorders (MAF: allele A, cases $=0.09$; controls $=0.12$ ) [37].

$\mathrm{Wu}$ et al. [38] performed an extensive search for transcriptional factor-binding sites and found that g.10403A>G $\mathrm{SNP}$ is located in a putative binding site for the transcription factor GATA-3. More importantly, only when the allele A 
TABLE 3: Correlation analysis of FOXP3 polymorphisms in relation to prognostic parameters in total BC sample and aggressive subtypes.

\begin{tabular}{lccccc}
\hline & Clinical outcomes & Total BC & \multicolumn{3}{c}{ Breast cancer subtypes $[p($ tau $)]$} \\
& $p(\operatorname{tau})$ & LB & HER2+ & TN \\
\hline & TNM staging & $0.173(\tau=0.11)$ & $0.608(\tau=0.08)$ & $0.835(\tau=-0.04)$ & $0.032(\tau=0.23)^{*}$ \\
g.10403A $>$ G & Tumor size & $0.633(\tau=-0.04)$ & $0.885(\tau=0.02)$ & $0.778(\tau=0.05)$ & $0.422(\tau=-0.10)$ \\
& Ki-67 & $0.270(\tau=0,11)$ & $0.837(\tau=0.04)$ & $0.019(\tau=0.47)^{*}$ & $0.536(\tau=0.08)$ \\
& Histological grade & $0.268(\tau=-0.10)$ & $0.846(\tau=-0.03)$ & $0.061(\tau=-0.36)$ & $0.909(\tau=-0.02)$ \\
& LP commitment & $0.298(\tau=0.09)$ & $0.337(\tau=0.15)$ & $0.175(\tau=0.255)$ & $0.934(\tau=-0.01)$ \\
\hline & TNM staging & $0.966(\tau=-0.003)$ & $0.894(\tau=0.02)$ & $0.167(\tau=0.23)$ & $0.084(\tau=-0.23)$ \\
g.8048A $>$ C & Tumor size & $0.166(\tau=0.10)$ & $0.403(\tau=0.11)$ & $0.912(\tau=-0.02)$ & $0.419(\tau=0.10)$ \\
& Ki-67 & $0.557(\tau=-0.06)$ & $0.28(\tau=-0.21)$ & $0.018(\tau=-0.47)^{*}$ & $0.708(\tau=0.05)$ \\
& Histological grade & $0.135(\tau=-0.15)$ & $0.754(\tau=0.06)$ & $0.026(\tau=0.39)^{*}$ & $0.927(\tau=0.01)$ \\
& LP commitment & $0.895(\tau=-0.01)$ & $0.662(\tau=-0.07)$ & $0.811(\tau=-0.05)$ & $0.913(\tau=0.02)$ \\
\hline
\end{tabular}

Kendall's tau test; *value of $p<0.05$ was considered statistically significant. BC: breast cancer; LB: Luminal B HER2+; HER2+: HER2-enriched; TN: triple-negative; LP: lymph node.

TABLE 4: FOXP3 haplotypes correlation analysis in relation to prognostic parameters in total BC sample and aggressive subtypes.

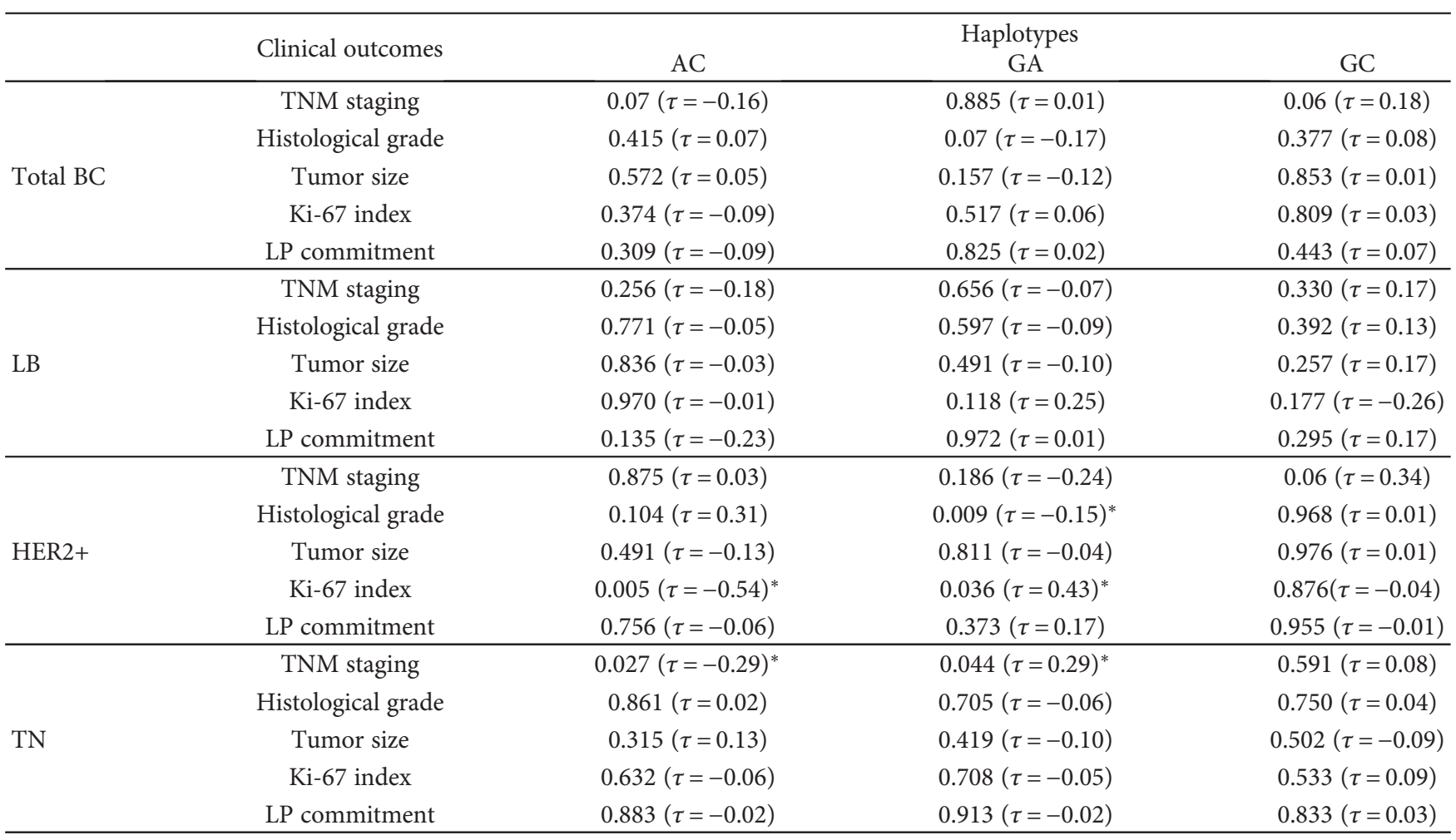

Kendall's tau test; *value of $p<0.05$ was considered statistically significant. LP: lymph node.

exists, this transcription factor can bind the promoter region of FOXP3. According to Wang et al. [39], defective function of both GATA-3 and FOXP3 itself led to ablation of Treg cells, suggesting that the combined function of these genes is essential for FOXP3 expression, highlighting the indispensable role of GATA-3 in regulating Treg cell function. In this context, the allele $\mathrm{A}$ of $\mathrm{g} .10403 \mathrm{~A}>\mathrm{G}$ may be associated with increased FOXP3 expression and, consequently, in the maintenance of Treg function, contributing to suppression of antitumor immune response. This fact may explain the positive association between this polymorphism and increased BC susceptibility.

In the present study, no association was found between g.8048A $>$ C and BC susceptibility, neither in general sample nor in different subtypes. Similar observations were made by Raskin et al. [40] in Israeli population (MAF: allele A, cases $=0.47$; controls $=0.47$ ), Zheng et al. [41] in Han Chinese population (MAF: allele A, cases $=0.19$; controls $=0.18$ ), and Jahan et al. [26] in Indian population (MAF: allele C, cases $=0.47$; controls $=0.44)$. Additionally, a meta-analysis 
performed by Jiang and Ruan [27] (MAF: allele A, cases = 0.37; controls $=0.34$ ) indicated that g.8048A $>C$ is not associated with $\mathrm{BC}$, but with susceptibility to hepatocellular carcinoma and nonsmall cell lung cancer.

No significant association between different FOXP3 haplotypes and $\mathrm{BC}$ susceptibility was observed, either in the general BC sample or in the different subtypes. To date, there are no studies relating the g.10403A $>\mathrm{G}$ and g.8048A $>\mathrm{C}$ haplotypes to BC susceptibility or clinical outcome, emphasizing that the present study is the first in the literature to describe this lack of association.

In addition, regarding $\mathrm{BC}$ prognosis, the present results showed a significant correlation of GG genotype (g.10403A>G) with higher proliferation index Ki-67 in HER2+ subtype and advanced TNM staging in TN subtype. To date, this is the first study that observed a correlation between g.10403A $>$ G polymorphism and BC prognostic parameters.

As previously discussed, the allele $G$ may be related to lower expression of FOXP3 due the lost binding site to GATA-3. Many studies have shown that, in BC, FOXP3 could be considered a tumor suppressor gene, conferring a better prognosis [42, 43].

Despite FOXP3, g.8048 A >C may not be playing a role in $\mathrm{BC}$ susceptibility in Brazilian women; we report a significant correlation of AA genotype with higher Ki-67 and lower histological grade in HER2+ subtype. No correlation of this polymorphism with prognostic parameters was found in TN subtype, which is in accordance with a previous study developed by our research group [44].

Like the GG genotype of g.10403A>G, the AA of g.8048A >C also appears to be related to FOXP3 lower expression. Shen et al. [45] observed that psoriatic patients with this genotype have reduced FOXP3 expression. These authors demonstrated that the $\mathrm{C}$ to A change causes binding loss to E47 and c-Myb transcription factors, leading to a defective FOXP3 gene transcription.

Furthermore, Jahan et al. [46] observed a highly significant association of AA (g.8048A $>$ C) with BC advanced stages (III and IV). In the present study, no correlation with tumor stage was found and, perhaps, this discrepant result is due to the BC subtypes studied. These authors did not stratify the BC sample and, probably, included subtypes of better prognosis, such as Luminal A and Luminal B HER2-, unlike the present study, which comprised only more aggressive BC subtypes.

In contrast, we found correlation of $\mathrm{AA}$ genotype (g.8048A >C) with higher histological grade. Ohara et al. [47] analyzed FOXP3 expression in breast tumor by qRTPCR and observed a significant correlation with higher histological grade. These authors attributed the correlation with worse prognosis to Treg infiltration. Other study using immunohistochemistry technique also showed association of FOXP3 expression by tumor cells with higher histological grade [48]. However, in this study, all tumor samples showed cytoplasmic or both cytoplasmic and nuclear FOXP3 expression, suggesting frequent deregulation of FOXP3 localization and failure to translocate to the nucleus in breast cancer cells and explaining the correlation with worse prognosis. In this context, the positive correlation of g.8048A $>C$ with histological grade may reflect the lower functional Treg infiltration in tumor bed.

Furthermore, significant correlations between FOXP3 haplotypes and prognostic parameters were found. The present study showed a correlation of AC with better prognosis, such as lower proliferation index and staging, in HER2+ and TN, respectively. As discussed above, the polymorphisms may affect the expression of gene, and, in this context, AC haplotype may be related with higher FOXP3 expression, possibly explaining the correlation with better prognosis, since this transcription factor is considered a BC tumor suppressor gene.

In conclusion, the present study showed, for the first time, a significant association of FOXP3 g.10403A>G with susceptibility and prognosis of aggressive $\mathrm{BC}$. Although the g.8048A >C may not be associated with BC susceptibility, significant correlations with clinical outcome were found. Furthermore, present study also showed, for the first time, different correlations regarding prognosis in LB, HER2+, and $\mathrm{TN}$, highlighting that the influence of allelic variants may depend on tumor subtype. Moreover, the dual role of FOXP3, participating in Treg cell development and function from one side and acting as a tumor modulator gene from other side should not be ignored.

\section{Ethical Approval}

All procedures performed in the studies involving human participants were in accordance with the ethical standards of the Institutional Human Research Ethics Committee of State University of Londrina and with the 1964 Helsinki declaration and its later amendments or comparable ethical standards.

\section{Consent}

Informed consent was obtained from all individual participants included in the study.

\section{Conflicts of Interest}

The authors declare that there are no conflicts of interest.

\section{Acknowledgments}

The authors would like to acknowledge the volunteers who made this study possible. This work was supported by Conselho Nacional de Desenvolvimento Científico e Tecnológico $(\mathrm{CNPq})$ and State University of Londrina Coordination for Post-Graduation (PROPPG-UEL). The authors wish to express their gratitude to Fundação Araucária do Paraná and Coordenação de Aperfeiçoamento de Pessoal de Nível Superior (CAPES).

\section{References}

[1] INCA, Estimate 2016: Cancer Incidence in Brazil, p. 51, Instituto Nacional de Câncer José Alencar Gomes da Silva, Rio de Janeiro, 2016. 
[2] B. Weigelt, A. Mackay, R. A'Hern et al., "Breast cancer molecular profiling with single sample predictors: a retrospective analysis," The Lancet Oncology, vol. 11, no. 4, pp. 339-349, 2010.

[3] A. P. Presson, N. K. Yoon, L. Bagryanova et al., "Protein expression based multimarker analysis of breast cancer samples," BMC Cancer, vol. 11, no. 230, pp. 1-14, 2011.

[4] A. Goldhirsch, E. P. Winer, A. S. Coates et al., "Personalizing the treatment of women with early breast cancer: highlights of the St Gallen International Expert Consensus on the Primary Therapy of Early Breast Cancer 2013," Annals of Oncology : Official Journal of the European Society for Medical Oncology 1 ESMO, vol. 24, no. 9, pp. 2206-2223, 2013.

[5] TCGA, "Comprehensive molecular portraits of human breast tumours," Nature, vol. 490, no. 7418, pp. 61-70, 2012.

[6] N. Cho, "Molecular subtypes and imaging phenotypes of breast cancer," Ultrasonography, vol. 35, no. 4, pp. 281-288, 2016.

[7] A. S. Coates, E. P. Winer, A. Goldhirsch et al., "Tailoring therapies-improving the management of early breast cancer: St Gallen International Expert Consensus on the Primary Therapy of Early Breast Cancer 2015," Annals of Oncology : Official Journal of the European Society for Medical Oncology / ESMO, vol. 26, no. 8, pp. 1533-1546, 2015.

[8] J. D. Fontenot, M. A. Gavin, and A. Y. Rudensky, "Foxp3 programs the development and function of CD4+CD25+ regulatory T cells," Nature Immunology, vol. 4, no. 4, pp. 330-336, 2003.

[9] G. J. Bates, S. B. Fox, C. Han et al., "Quantification of regulatory T cells enables the identification of high-risk breast cancer patients and those at risk of late relapse," Journal of Clinical Oncology: Official Journal of the American Society of Clinical Oncology, vol. 24, no. 34, pp. 5373-5380, 2006.

[10] S. Ladoire, L. Arnould, L. Apetoh et al., "Pathologic complete response to neoadjuvant chemotherapy of breast carcinoma is associated with the disappearance of tumor-infiltrating foxp3+ regulatory T cells," Clinical Cancer Research : An Official Journal of the American Association for Cancer Research, vol. 14, no. 8, pp. 2413-2420, 2008.

[11] N. Leffers, M. J. Gooden, R. A. de Jong et al., "Prognostic significance of tumor-infiltrating T-lymphocytes in primary and metastatic lesions of advanced stage ovarian cancer," Cancer Immunology, Immunotherapy : CII, vol. 58, no. 3, pp. 449-459, 2009.

[12] S. Hinz, L. Pagerols-Raluy, H. H. Oberg et al., "Foxp3 expression in pancreatic carcinoma cells as a novel mechanism of immune evasion in cancer," Cancer Research, vol. 67, no. 17, pp. 8344-8350, 2007.

[13] L. M. Ebert, B. S. Tan, J. Browning et al., “The regulatory T cellassociated transcription factor FoxP3 is expressed by tumor cells," Cancer Research, vol. 68, no. 8, pp. 3001-3009, 2008.

[14] S. Ladoire, L. Arnould, G. Mignot et al., "Presence of Foxp3 expression in tumor cells predicts better survival in HER2-overexpressing breast cancer patients treated with neoadjuvant chemotherapy," Breast Cancer Research and Treatment, vol. 125, no. 1, pp. 65-72, 2011.

[15] L. F. Lopes, R. L. Guembarovski, A. L. Guembarovski et al., "FOXP3 transcription factor: a candidate marker for susceptibility and prognosis in triple negative breast cancer," BioMed Research International, vol. 2014, Article ID 341654, 7 pages, 2014.
[16] A. Merlo, P. Casalini, M. L. Carcangiu et al., "FOXP3 expression and overall survival in breast cancer," Journal of Clinical Oncology : Official Journal of the American Society of Clinical Oncology, vol. 27, no. 11, pp. 17461752, 2009.

[17] T. Zuo, R. Liu, H. Zhang et al., "FOXP3 is a novel transcriptional repressor for the breast cancer oncogene SKP2," The Journal of Clinical Investigation, vol. 117, no. 12, pp. 37653773, 2007.

[18] T. Zuo, L. Wang, C. Morrison et al., "FOXP3 is an X-linked breast cancer suppressor gene and an important repressor of the HER-2/ErbB2 oncogene," Cell, vol. 129, no. 7, pp. 12751286, 2007.

[19] M. Fazelzadeh Haghighi, M. Ali Ghayumi, F. Behzadnia, and N. Erfani, "Investigation of FOXP3 genetic variations at positions $-2383 \mathrm{C} / \mathrm{T}$ and IVS9+459 T/C in southern Iranian patients with lung carcinoma," Iranian Journal of Basic Medical Sciences, vol. 18, no. 5, pp. 465-471, 2015.

[20] E. Fodor, E. Garaczi, H. Polyanka, A. Koreck, L. Kemeny, and M. Szell, "The rs3761548 polymorphism of FOXP3 is a protective genetic factor against allergic rhinitis in the Hungarian female population," Human Immunology, vol. 72, no. 10, pp. 926-929, 2011.

[21] G. M. Andre, C. P. Barbosa, J. S. Teles, F. L. Vilarino, D. M. Christofolini, and B. Bianco, "Analysis of FOXP3 polymorphisms in infertile women with and without endometriosis," Fertility and Sterility, vol. 95, no. 7, pp. 2223-2227, 2011.

[22] P. M. Ozawa, C. B. Ariza, R. Losi-Guembarovski et al., "Wilms' tumor susceptibility: possible involvement of FOXP3 and CXCL12 genes," Molecular and Cellular Pediatrics, vol. 3, no. 1, p. 36, 2016.

[23] Y. Chen, H. Zhang, W. Liao et al., "FOXP3 gene polymorphism is associated with hepatitis B-related hepatocellular carcinoma in China," Journal of Experimental \& Clinical Cancer Research: CR, vol. 32, no. 1, pp. 32-39, 2013.

[24] L. Chen, Q. Yu, B. Liu, and L. Zhu, “Association of FoxP3 rs3761548 polymorphism with susceptibility to colorectal cancer in the Chinese population," Medical Oncology, vol. 31, no. 12 , p. 374, 2014.

[25] Y. Q. He, Q. Bo, W. Yong, Z. X. Qiu, Y. L. Li, and W. M. Li, "FoxP3 genetic variants and risk of non-small cell lung cancer in the Chinese Han population," Gene, vol. 531, no. 2, pp. 422-425, 2013.

[26] P. Jahan, V. R. Ramachander, G. Maruthi, S. Nalini, K. P. Latha, and T. S. Murthy, "Foxp3 promoter polymorphism (rs3761548) in breast cancer progression: a study from India," Tumour Biology: The Journal of the International Society for Oncodevelopmental Biology and Medicine, vol. 35, no. 4, pp. 3785-3791, 2014.

[27] L. L. Jiang and L. W. Ruan, "Association between FOXP3 promoter polymorphisms and cancer risk: a meta-analysis," Oncology Letters, vol. 8, no. 6, pp. 2795-2799, 2014.

[28] L. H. Sobin, M. K. Gospodarowicz, and C. Wittekind, TNM Classification of Malignant Tumours, 2009.

[29] A. Paradowska-Gorycka, M. Jurkowska, A. Felis-Giemza et al., "Genetic polymorphisms of Foxp3 in patients with rheumatoid arthritis," The Journal of Rheumatology, vol. 42, no. 2, pp. 170-180, 2015.

[30] M. Stephens and P. Scheet, "Accounting for decay of linkage disequilibrium in haplotype inference and missing-data 
imputation," American Journal of Human Genetics, vol. 76, no. 3, pp. 449-462, 2005.

[31] M. Stephens, N. J. Smith, and P. Donnelly, "A new statistical method for haplotype reconstruction from population data," American Journal of Human Genetics, vol. 68, no. 4, pp. 978989, 2001.

[32] M. J. Machiela and S. J. Chanock, "LDlink: a web-based application for exploring population-specific haplotype structure and linking correlated alleles of possible functional variants,” Bioinformatics, vol. 31, no. 21, pp. 3555-3557, 2015.

[33] Q. H. Song, Z. Shen, X. J. Xing et al., "An association study of single nucleotide polymorphisms of the FOXP3 intron-1 and the risk of psoriasis vulgaris," Indian Journal of Biochemistry \& Biophysics, vol. 49, no. 1, pp. 25-35, 2012.

[34] P. Song, X. W. Wang, H. X. Li et al., "Association between FOXP3 polymorphisms and vitiligo in a Han Chinese population," The British Journal of Dermatology, vol. 169, no. 3, pp. 571-578, 2013.

[35] Z. G. Wu, Z. S. You, C. Zhang et al., "Study on association of functional polymorphisms in Foxp3 gene with the susceptibility to unexplained recurrent spontaneous abortion," Zhonghua Fu Chan Ke Za Zhi, vol. 46, no. 10, pp. 763-768, 2011.

[36] D. Saxena, M. K. Misra, F. Parveen, S. R. Phadke, and S. Agrawal, "The transcription factor Forkhead box P3 gene variants affect idiopathic recurrent pregnancy loss," Placenta, vol. 36, no. 2, pp. 226-231, 2015.

[37] M. R. Safari, S. Ghafouri-Fard, R. Noroozi et al., "FOXP3 gene variations and susceptibility to autism: a case-control study," Gene, vol. 596, no. 1, pp. 119-122, 2017.

[38] Z. Wu, Z. You, C. Zhang et al., “Association between functional polymorphisms of Foxp3 gene and the occurrence of unexplained recurrent spontaneous abortion in a Chinese Han population," Clinical \& Developmental Immunology, vol. 2012, Article ID 896458, 7 pages, 2012.

[39] Y. Wang, M. A. Su, and Y. Y. Wan, "An essential role of the transcription factor GATA-3 for the function of regulatory T cells," Immunity, vol. 35, no. 3, pp. 337-348, 2011.

[40] L. Raskin, G. Rennert, and S. B. Gruber, "FOXP3 germline polymorphisms are not associated with risk of breast cancer," Cancer Genetics and Cytogenetics, vol. 190, no. 1, pp. 40-42, 2009.

[41] J. Zheng, J. Deng, L. Jiang et al., "Heterozygous genetic variations of FOXP3 in Xp11.23 elevate breast cancer risk in Chinese population via skewed X-chromosome inactivation," Human Mutation, vol. 34, no. 4, pp. 619-628, 2013.

[42] C. Zhang, Y. Xu, Q. Hao et al., "FOXP3 suppresses breast cancer metastasis through downregulation of CD44," International Journal of Cancer, vol. 137, no. 6, pp. 1279-1290, 2015.

[43] S. Douglass, A. P. Meeson, D. Overbeck-Zubrzycka et al., "Breast cancer metastasis: demonstration that FOXP3 regulates CXCR4 expression and the response to CXCL12," The Journal of Pathology, vol. 234, no. 1, pp. 74-85, 2014.

[44] L. F. Lopes, R. L. Guembarovski, A. L. Guembarovski et al., "FOXP3 transcription factor: a candidate marker for susceptibility and prognosis in triple negative breast cancer," BioMed Research International, vol. 2014, Article ID 341654, 7 pages, 2014.

[45] Z. Shen, L. Chen, F. Hao, G. Wang, and Y. Liu, "Intron-1 rs3761548 is related to the defective transcription of Foxp3 in psoriasis through abrogating E47/c-Myb binding,"
Journal of Cellular and Molecular Medicine, vol. 14, no. 1-2, pp. 226-241, 2010.

[46] P. Jahan, R. Cheruvu, S. Tippisetty, P. L. Komaravalli, V. Valluri, and M. Ishaq, "Association of FOXP3 (rs3761548) promoter polymorphism with nondermatomal vitiligo: a study from India," Journal of the American Academy of Dermatology, vol. 69, no. 2, pp. 262-266, 2013.

[47] M. Ohara, Y. Yamaguchi, K. Matsuura, S. Murakami, K. Arihiro, and M. Okada, "Possible involvement of regulatory $\mathrm{T}$ cells in tumor onset and progression in primary breast cancer," Cancer Immunology, Immunotherapy : CII, vol. 58, no. 3, pp. 441-447, 2009.

[48] M. H. Kim, J. S. Koo, and S. Lee, "FOXP3 expression is related to high Ki-67 index and poor prognosis in lymph nodepositive breast cancer patients," Oncology, vol. 85, no. 2, pp. 128-136, 2013. 


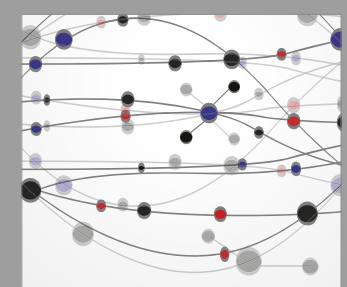

The Scientific World Journal
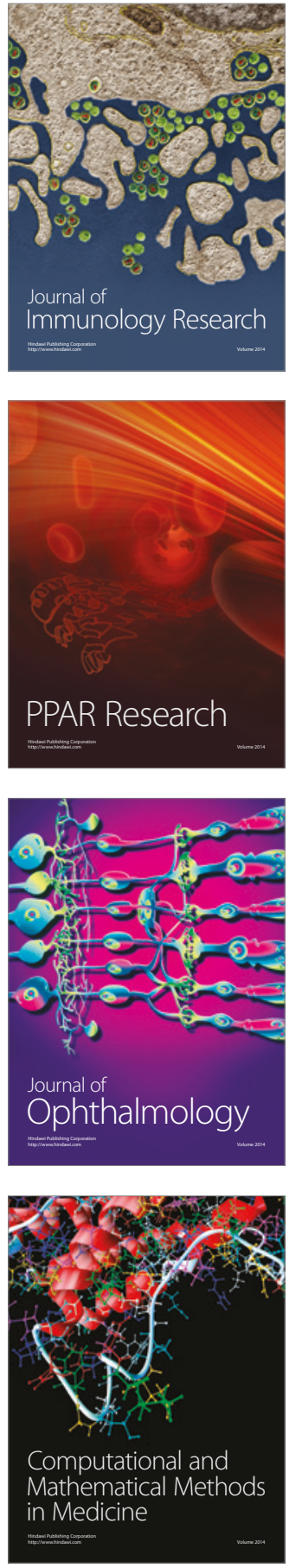

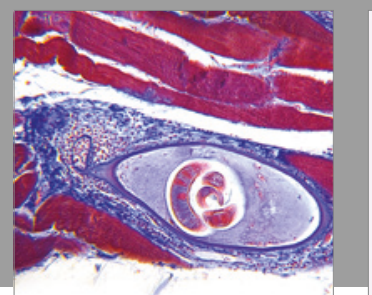

Gastroenterology Research and Practice
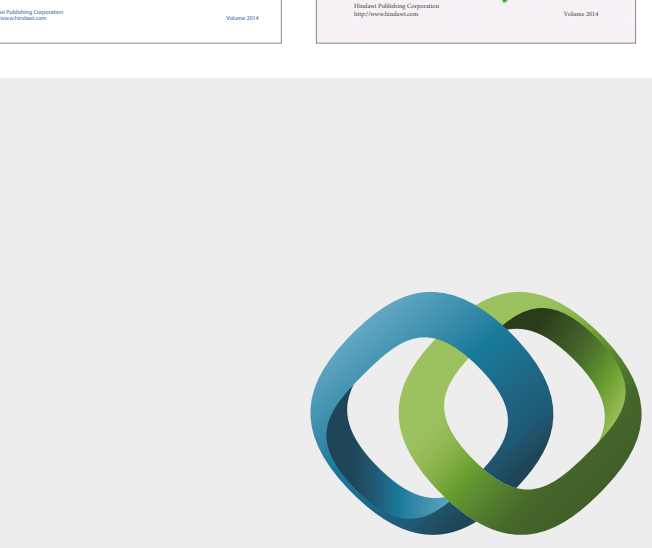

\section{Hindawi}

Submit your manuscripts at

https://www.hindawi.com
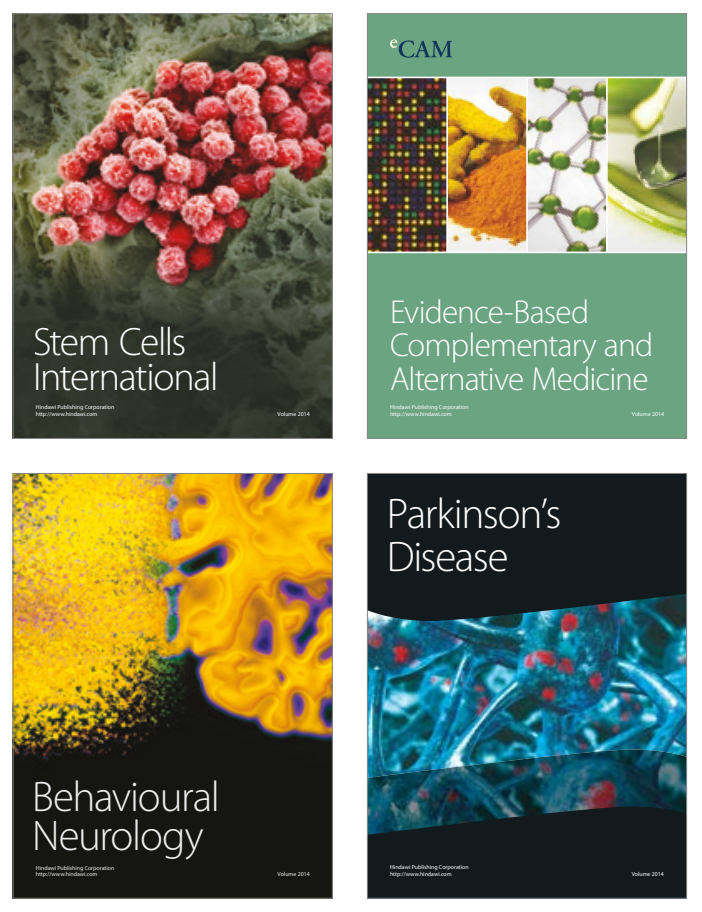
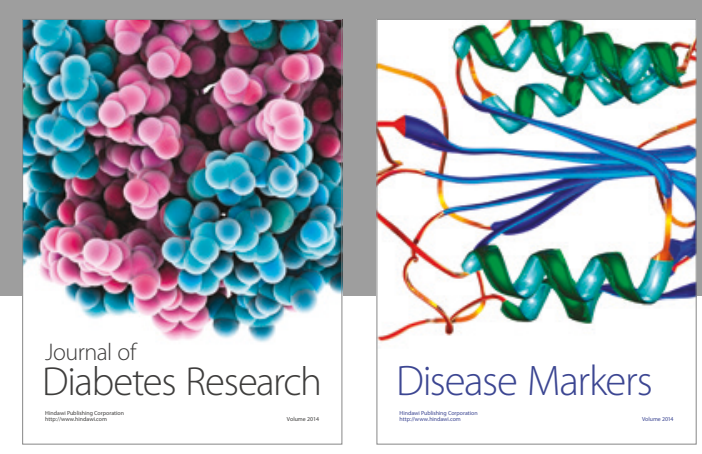

Disease Markers
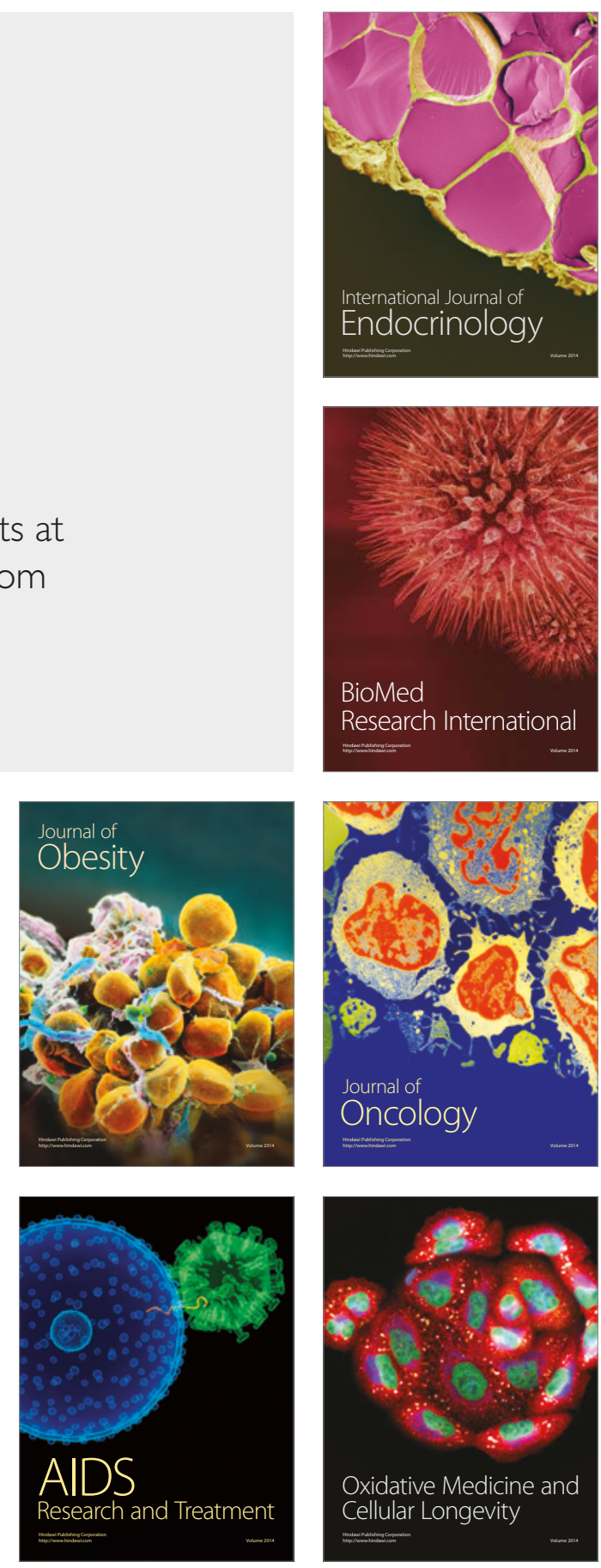